\title{
Impacts of Vertical Structure of Large-Scale Vertical Motion in Tropical Climate: Moist Static Energy Framework ${ }^{\mathscr{O}}$
}

\author{
HiEN XUAN BUI \\ Earth System Science Program, Taiwan International Graduate Program, Research Center for Environmental \\ Changes, Academia Sinica, Taipei, and Department of Atmospheric Sciences, National Central University, \\ Taoyuan City, Taiwan \\ JIA-YUH YU \\ Department of Atmospheric Sciences, National Central University, Taoyuan City, Taiwan \\ CHIA CHOU \\ Research Center for Environmental Changes, Academia Sinica, and Department of Atmospheric Sciences, \\ National Taiwan University, Taipei, Taiwan
}

(Manuscript received 20 January 2016, in final form 27 July 2016)

\begin{abstract}
Interactions between cumulus convection and its large-scale environment have been recognized as crucial to the understanding of tropical climate and its variability. In this study, the moist static energy (MSE) budget is employed to investigate the potential impact of the vertical structure of large-scale vertical motion in tropical climate based on results from both reanalysis data and model simulation. Two domains are selected over the western and eastern Pacific with vertical motion profiles that are dominated by top-heavy and bottom-heavy structures, respectively. The bottom-heavy structure is climatologically associated with more shallow convection, while the top-heavy structure is related to more deep convection. The column-integrated vertical MSE advection of top-heavy vertical motion is positive, while that of bottom-heavy vertical motion tends to be negative. Controlling factors responsible for the above vertical MSE advection contrast are discussed based on a simple decomposition of the MSE budget equation. It was found that the sign of vertical MSE advection is determined mainly by the vertical moisture transport, the magnitude of which is very sensitive to the structure of vertical motion. A top-heavy (bottom heavy) structure of vertical motion favors an export (import) of MSE and a positive (negative) value of the vertical MSE advection.
\end{abstract}

\section{Introduction}

The efficiency of precipitation production in a convective cloud depends largely on how high convection can develop along the vertical direction. For example, heavy rainfall events tend to occur in regions of deep convection where the cloud top can often grow up to the tropopause,

Supplemental information related to this paper is available at the Journals Online website: http://dx.doi.org/10.1175/ JAS-D-16-0031.s1.

Corresponding author address: Dr. Jia-Yuh Yu, Department of Atmospheric Sciences, National Central University, 300 Zhongda Road, Zhongli District, Taoyuan City 32001, Taiwan.

E-mail: jiayuh@atm.ncu.edu.tw such as the western Pacific warm pool. On the other hand, light rain events are generally associated with shallow convection that often occurs in regions of trade wind inversion (e.g., the eastern regions of the subtropical oceans). While shallow convection involves, on average, less significant latent heating and moisture sink compared to deep convection, it plays an important role in transporting heat and moisture from the boundary layer to the lower troposphere that might precondition the development of deep convection later (Neggers et al. 2007).

Shallow convection has become an important research topic since the first studies on scale analysis of shallow convection in the early 1960s (Ogura and Phillips 1962; Lilly 1968). Observational studies based on satellite-radar remote sensing have shown that changes of temperature and moisture are coherent with detraining air at the 
cumulus cloud top (Takayabu et al. 2010). Modeling studies have also shown that the vertical transport of heat and moisture by shallow convection strongly influences the boundary layer depth, temperature, humidity, cloud cover, and wind as a result of air from the surface being mixed in the free troposphere (Bretherton et al. 2004), and these effects will in turn provide a favorable environment for the development of deep convection (Wu et al. 2009; Waite and Khouider 2010; Yano and Plant 2012; Wang 2014). Since cumulus convection occurs on much smaller scales than data gathered by conventional meteorological networks, its effects on the large-scale circulation cannot be directly measured. Instead, these effects are often inferred indirectly from heat and moisture budgets of the larger-scale circulation system (Yanai and Johnson 1993). In this study, we employ a moist static energy (MSE) budget to examine the interaction between cumulus convection and its associated largescale environment, focusing on the time-mean tropical climate. In deep convective regions-referring to regions dominated by a top-heavy structure of vertical motion when averaged over climatic time scales-the column-integrated vertical MSE advection has a positive value, which means that the MSE is exported from the convective region to its surrounding environment in order to stabilize the air column. In shallow convective regions (referring to the regions dominated by a bottom-heavy structure of vertical motion when averaged over climatic time scales), the columnintegrated vertical MSE advection is generally negative, which implies an import of MSE and a destabilization of the air column.

To provide a simple model framework for the timemean tropical convergence, the quantity termed "gross moist stability" (GMS) was first defined by Neelin and Held (1987) in a two-layer model based on the MSE budget, and later it was redefined in a continuously stratified atmosphere subject to the Betts-Miller convective quasi-equilibrium constraints by Neelin and Yu (1994), Yu and Neelin (1994, 1997), and Yu et al. (1998). In this type of model, GMS serves as a measurement of the effective static stability in a large-scale dynamic framework where the effect of water vapor is explicitly considered. Physically, the magnitude of the GMS represent the efficiency of an air column in exporting MSE in the presence of large-scale vertical motions. The magnitude of GMS is found to be small and positive in the tropics, and its size is sensitive to the upper bound of vertical integration and profiles of temperature and moisture (Yu et al. 1998). The same treatment of GMS is also used in the Quasi-Equilibrium Tropical Circulation Model, version 1, (QTCM1) by Neelin and Zeng (2000).
A different approach proposed by Raymond et al. (2009) is to normalize the GMS by the moisture convergence such that estimates of GMS magnitude become less sensitive to the choice of cloud-top height. One can also include the radiative feedback and surface fluxes in the GMS to obtain an effective GMS, which was found to be important for the evolution of the tropical Madden-Julian oscillation (Maloney et al. 2010; Hannah and Maloney 2014).

To address the role of different types of convection, Back and Bretherton (2006) considered the geographical variability of cumulus convection between the western and eastern Pacific. By calculating the MSE budget, they showed that a bottom-heavy structure in the eastern Pacific is indeed importing, not exporting, MSE into convective regions. To investigate the importance of cloud-top height (or, practically, the upper bound of integration) on the changes of GMS, Chou et al. (2013) estimated the magnitude of GMS with varying cloud-top levels. Their study showed that the sign of GMS is very sensitive to the choice of the upper bound of integration. A positive value of GMS generally occurs when a higher upper bound of integration is chosen (e.g., above $300 \mathrm{hPa}$ ). On the other hand, a lower upper bound of integration (e.g., below $450 \mathrm{hPa}$ ) often produces a negative value of GMS.

In this study, we propose a simple approach for investigating the impact of the vertical structure of the large-scale vertical motion in tropical climate. We utilize the MSE framework, especially using the columnintegrated vertical MSE advection-an equivalent of GMS - to contrast the very different roles played by topheavy and bottom-heavy vertical motions in venting MSE and to explore the possible causes responsible for such differences. To illustrate this approach, we apply it to both reanalysis and model simulation data. The European Centre for Medium-Range Weather Forecasts (ECMWF) interim reanalysis (ERA-Interim) is used here instead of the ERA-40 used in Back and Bretherton (2006) and Peters et al. (2008). Using a much improved atmospheric model and assimilation system, the ERA-Interim is more skillful than the ERA-40, especially for the moisture and precipitation fields over oceans (Dee et al. 2011). The Community Earth System Model (CESM) simulation outputs are also analyzed as a comparison. We describe the data used and methodology in section 2, discuss the differences in venting MSE between the western and eastern Pacific in section 3 , and give a detailed examination and discussion of possible causes responsible for the above differences in section 4. Major findings are summarized and discussed in section 5 . 


\section{Data and methods}

\section{a. Data}

To examine the controlling factors of the columnintegrated vertical MSE advection between the west and east Pacific, two datasets are used. The first one is taken from the ERA-Interim (Dee et al. 2011; http://apps.ecmwf.int/datasets/). Daily data during the period from 1998 to 2007 are used with a horizontal resolution of $1.5^{\circ} \times 1.5^{\circ}$ at 37 pressure levels.

Model data are taken from the fully coupled simulation of the CESM, version 1.0.3, with a spatial resolution of $1^{\circ} \times 1^{\circ}$ and temporal resolution of daily outputs. The simulation was run for almost $240 \mathrm{yr}$, but only $30 \mathrm{yr}$ of output data (including El Niño, La Niña, and normal years) were used for analysis. For the precipitation, we use the satellite-retrieved precipitation data from the Tropical Rainfall Measuring Mission (TRMM) (version 3B42) during the period of 1998-2007. The original spatial resolution for the TRMM precipitation product is $0.25^{\circ} \times 0.25^{\circ}$, but it was regridded into $1^{\circ} \times 1^{\circ}$ to be consistent with the CESM outputs.

\section{b. Moist static energy budget}

Following Chou et al. (2009a) and Chou et al. (2009b), the time-mean, vertically integrated MSE and moisture budgets can be written as

$$
\left\langle\omega \partial_{p} h\right\rangle=-\langle\mathbf{v} \cdot \nabla(q+T+g z)\rangle+F^{\mathrm{net}}
$$

and

$$
\left\langle\omega \partial_{p} q\right\rangle+\langle\mathbf{v} \cdot \nabla q\rangle=E-P .
$$

Most of the notations in (1) and (2) are conventional: $E$ is evaporation, $P$ is precipitation, and $\mathbf{v}$ is the horizontal wind vector; temperature $T$ can be expressed in energy units $\left(\mathrm{J} \mathrm{kg}^{-1}\right)$ by absorbing the heat capacity at constant pressure $C_{p}$. The specific humidity $q$, for consistency, can also be expressed in energy units $\left(\mathrm{J} \mathrm{kg}^{-1}\right)$ by absorbing the latent heat per unit mass $L$. The moist static energy $h=$ $q+s$ is physically similar to the equivalent potential temperature $\theta_{e}$, but the former is subject to the hydrostatic approximation (Ma et al. 2015). Dry static energy is $s=$ $T+g z$, with $g$ denoting gravity and $z$ being the height. The angle brackets \langle\rangle denotes a mass integration through the troposphere, from $1000 \mathrm{hPa}$ to the upper bound, typically the tropopause pressure. The upper bound of integration is initially fixed at $100 \mathrm{hPa}$ but is allowed to vary later to examine the sensitivity of vertical MSE advection to the choices of the upper bound of integration. The left-hand side term $\left\langle\omega \partial_{p} h\right\rangle$ represents the export-import of MSE by large-scale vertical motions and has a value that is highly associated with the GMS (Raymond et al. 2009). On the right-hand side, the first term $-\langle\mathbf{v} \cdot \nabla(q+T+g z)\rangle$ describes the horizontal MSE advection, and the second term $F^{\text {net }}$ is the net fluxes into the air column, defined as

$$
F^{\text {net }}=S_{t}^{\downarrow}-S_{t}^{\uparrow}-S_{s}^{\downarrow}+S_{s}^{\uparrow}-R_{t}^{\uparrow}-R_{s}^{\downarrow}+R_{s}^{\uparrow}+E+H .
$$

These fluxes include shortwave radiation flux $S$, longwave radiation flux $R$, surface evaporation $E$, and sensible heat flux $H$. Superscripts $\downarrow$ and $\uparrow$ indicate downward and upward fluxes, respectively, while subscripts $t$ and $s$ represent fluxes at the top of the atmosphere and surface, respectively.

\section{c. Singular value decomposition analysis}

To examine the connection between MSE and $\omega$ profiles in the day-to-day changes, we use the singular value decomposition (SVD) method to extract the dominant coupled modes of these two fields and their associated singular value time series. Further details on SVD analysis in climate research can be found in Bretherton et al. (1992), Wallace et al. (1992), and many others [see also Newman and Sardeshmukh 1995; Cherry 1996, 1997]. In this study, the SVD calculation is carried out with the MSE profile as the left field and the $\omega$ profile as the right field for each region. The results are then standardized for ease of discussion.

The expansion coefficients time series obtained by projecting the singular vectors onto the original data fields depicts the temporal correlations of the vertical profiles. The correlation coefficient between these time series for each mode quantifies the strength of the coupled structure described by that mode. The heterogeneous correlation profiles are calculated between a time series of a field and the other field to indicate the key regions accounting for the covariability. In other words, it shows how well the grid points in the first field can be predicted from the second field. The homogeneous correlation structure, on the other hand, is a useful indicator of the region of the covarying part of each field.

\section{Two modes of tropical large-scale vertical motion}

Figure 1 shows the climatological vertical MSE advection, $\left\langle\omega \partial_{p} h\right\rangle$, and precipitation over the tropical Pacific averaged from the daily mean fields. The white curves enclose rainy regions with annual-mean rainfall intensity exceeding $4 \mathrm{~mm}_{\text {day }}{ }^{-1}$. Here, we only focus on oceanic regions to avoid complications caused by the topographical effect. The total precipitation amount seems to be larger in the eastern Pacific intertropical convergence zone 
(a) Precipitation CESM
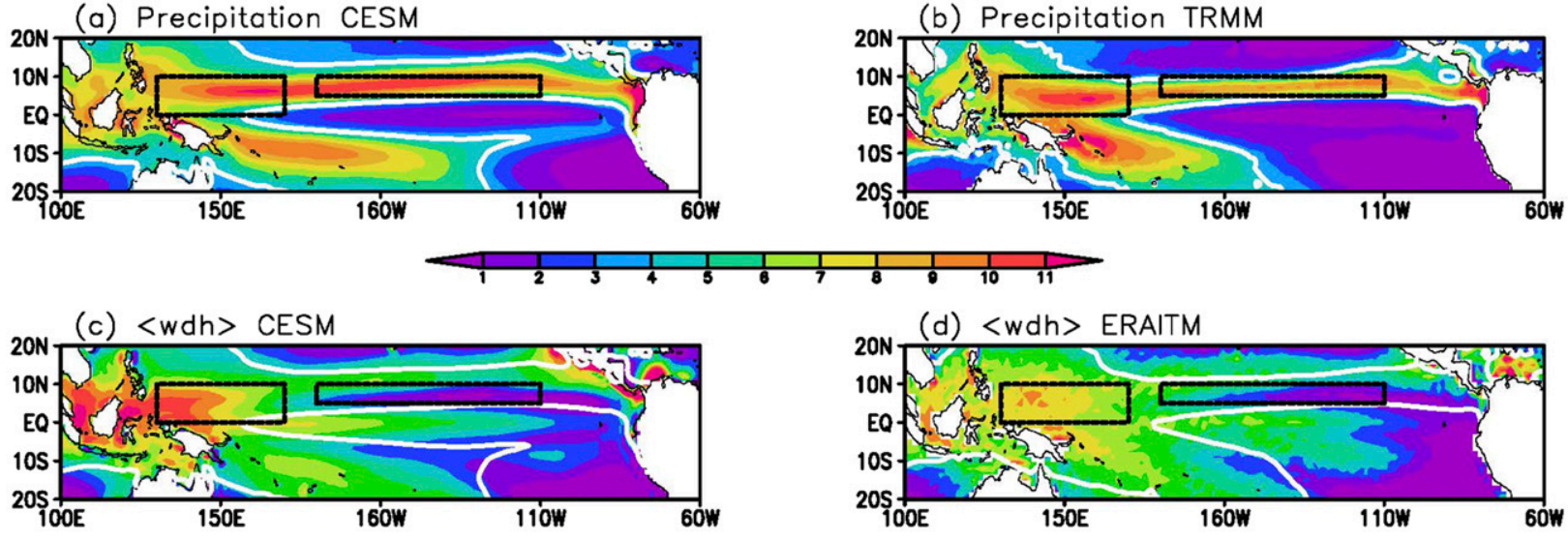

FIG. 1. Climatology of (a),(b) precipitation $\left(\mathrm{mm} \mathrm{day}^{1}\right)$ and (c),(d) column-integrated MSE vertical advection $\left(\mathrm{W} \mathrm{m}^{-2}\right)$ from (a),(c) the CESM and (d) the ERA-Interim. The precipitation in (b) is from TRMM. The boxes in each panel show the analysis domains for the western Pacific ITCZ $\left(0^{\circ}-10^{\circ} \mathrm{N}, 130^{\circ}-170^{\circ} \mathrm{E}\right.$; left $)$ and the eastern Pacific ITCZ $\left(5^{\circ}-10^{\circ} \mathrm{N}, 180^{\circ}-110^{\circ} \mathrm{W}\right.$; right). The white curve is $4 \mathrm{~mm} \mathrm{day}^{-1}$ of precipitation.

(ITCZ) than that in the western Pacific, as shown by the CESM simulations (see Fig. 1a). This feature is not observed in TRMM observations (see Fig. 1b), implying the model's precipitation bias in CESM. We note that vertical MSE advection is positive in the western Pacific ITCZ but mostly negative in the eastern Pacific ITCZ despite its pattern being in general similar to the mean precipitation. When compared to the ERA-Interim, the magnitude of vertical MSE advection in CESM is a little stronger, in particular, over the western Pacific warm pool.

Motivated by the east-west contrast of the vertical motion in Back and Bretherton (2006), we choose two boxes of approximately equivalent area over the western Pacific $\left(0^{\circ}-10^{\circ} \mathrm{N}, 130^{\circ}-170^{\circ} \mathrm{E}\right)$ and the eastern Pacific $\left(5^{\circ}-10^{\circ} \mathrm{N}, 180^{\circ}-110^{\circ} \mathrm{W}\right)$ ITCZs to represent the domains dominated by top-heavy and bottom-heavy structures of vertical motion, respectively (see Figs. S2 and S3 and the associated discussion in the supplemental material for justification of the domain selection). The anomalous MSE budget analysis discussed in section 4 will help distinguish the controlling factors responsible for the very different estimates of vertical MSE advection between the western and eastern Pacific ITCZs.

\section{a. Vertical motion and MSE profiles}

As shown in Fig. 2, the vertical motion profiles suggest two significant modes with different peaks of vertical motion in the tropics. The first mode, typically occurring over the western Pacific ITCZ, shows a top-heavy structure with the peak ascending motion at around $400 \mathrm{hPa}$; while the second mode, typically occurring over the eastern Pacific ITCZ, has a bottom-heavy structure with the peak ascending motion at around $850 \mathrm{hPa}$ (see Fig. 2a), generally consistent with Back and Bretherton (2006). The top-heavy profile of vertical motion over the western Pacific ITCZ indicates a higher frequency of deep convection in this domain, which is in response to the high sea surface temperature (SST) and the lower atmospheric stability to effectively release the MSE accumulated by the large-scale flows (Neelin and Held 1987). Unlike the western Pacific, the SST in the eastern Pacific is relatively cool, and the lower atmosphere is often capped by an inversion layer that stops the convection from going deep. In this region, it is the strong SST gradients that drive the boundary layer convergence (Lindzen and Nigam 1987) to produce as much precipitation as in the western Pacific ITCZ. As mentioned in Back and Bretherton (2006) and later in Sobel (2007), strong boundary layer convergence favors a bottom-heavy structure of vertical motion, with a peak ascending motion near the boundary layer top and a divergent return flow near the freezing level. For the entire tropics $\left(20^{\circ} \mathrm{S}-20^{\circ} \mathrm{N}\right)$, the averaged shape of $\omega$ shows a slightly bottom-heavy structure with a much smaller magnitude, implying the coexistence of ascending and descending motions when averaged over a much larger domain (not shown).

The corresponding vertical profile of MSE is shown in Fig. 2b. In general, the western Pacific ITCZ shows a slightly greater MSE throughout the troposphere compared to the eastern Pacific ITCZ, although the difference seems to be less significant in the CESM simulation compared with that in the ERA-Interim. The MSE difference between CESM and ERA-Interim is larger in the upper and lower troposphere, possibly because of biases in temperature and moisture simulation in the CESM model simulation. A 

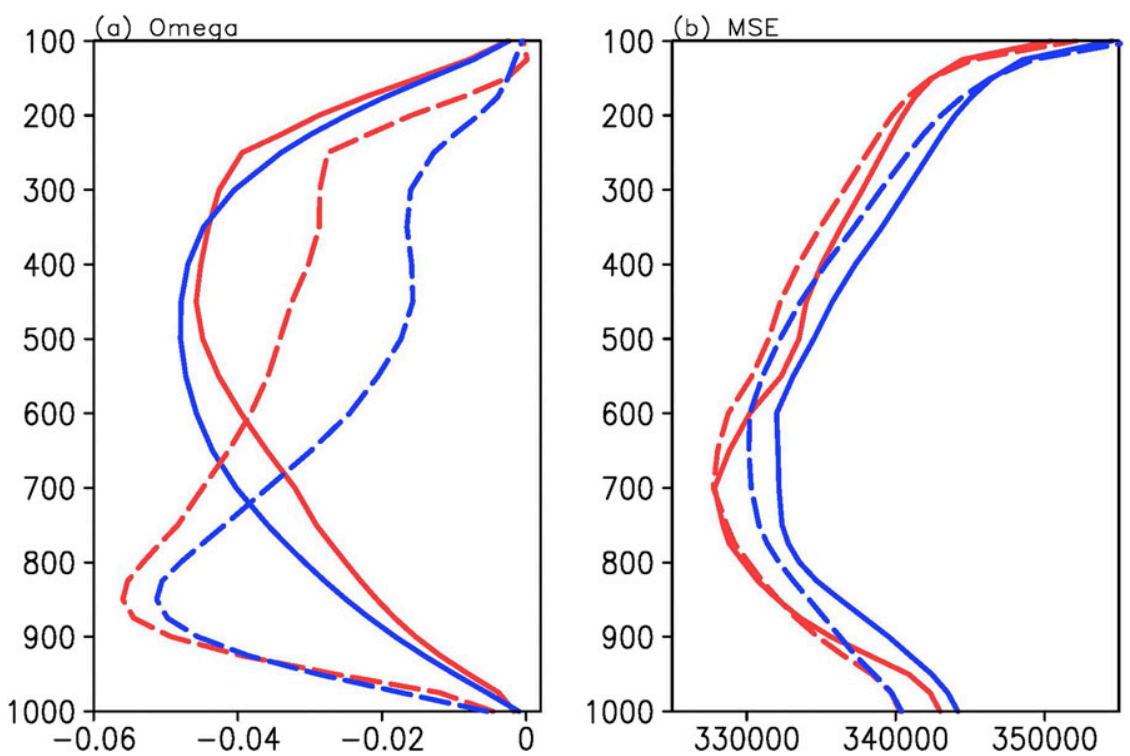

FIG. 2. Vertical profiles of (a) $\omega\left(\mathrm{Pa} \mathrm{s}^{-1}\right)$ and (b) $\mathrm{MSE}\left(\mathrm{W} \mathrm{m}^{-2}\right)$ over the western Pacific (solid) and eastern Pacific (dashed) from the CESM (red) and ERA-Interim (blue).

detailed examination of the correlation between MSE and $\omega$ profiles is presented in the appendix to provide additional information on the annual cycle of these variables.

\section{b. Column-integrated vertical MSE advection}

Figure 3a shows the term budget of vertical MSE advection averaged over the two target domains. In the western Pacific ITCZ, the detrainment of deep convection often occurs at the upper troposphere, where the mean MSE of detraining air is higher than that of the boundary layer converging air. In the eastern Pacific ITCZ, on the other hand, the detrainment of shallower convection generally starts at relatively lower levels, where the MSE of detraining air is lower than that of the boundary layer converging air. As a result, the vertical MSE advection associated with a top-heavy structure of vertical motion is positive, while that associated with a bottom-heavy structure of vertical motion is negative. The horizontal MSE advection is generally negative, implying the horizontal intrusion of cold and dry air into the atmospheric column in the presence of convection. In the eastern Pacific ITCZ, the magnitude of the horizontal MSE advection is much larger compared to that in the western Pacific ITCZ because of strong SST gradients in this region. The net flux term is positive in both regions, with a greater magnitude in the western Pacific ITCZ as a result of warmer SSTs in this region compared with the eastern Pacific ITCZ. We note that the residual term is more negative in both the western Pacific and the eastern Pacific in the ERA-Interim, possibly associated with the inconsistencies between the MSE advection and the diabatic MSE source (Back and Bretherton 2006) and/or the inconsistencies between model and observational updates. For the CESM analysis, the large time-mean MSE budget residual could be rationalized as the errors of coordinate transfer, for instance, from the sigma $\sigma$ to pressure $p$ coordinate (Chen et al. 2016).
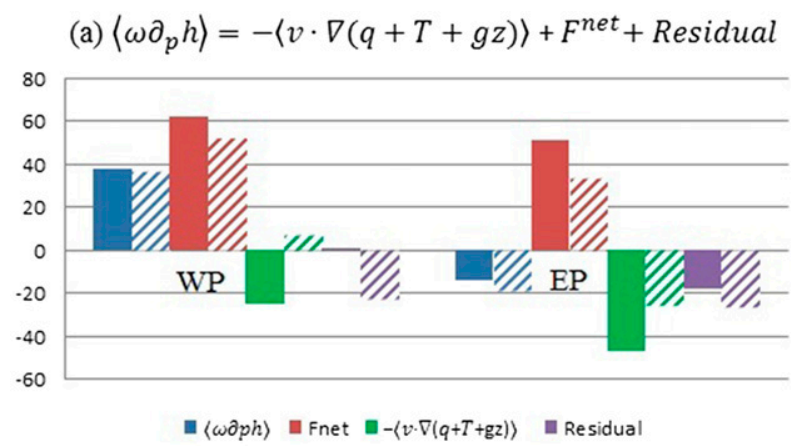

(b) $\left\langle\omega \partial_{p} h\right\rangle=\left\langle\omega \partial_{p} s\right\rangle+\left\langle\omega \partial_{p} q\right\rangle$

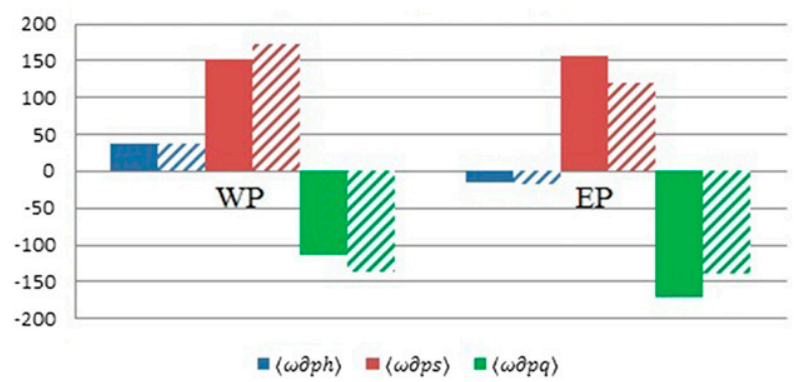

FIG. 3. The averages of the (a) column-integrated MSE budgets (W m ${ }^{-2}$, see legend below panel) and (b) $\left\langle\omega \partial_{p} h\right\rangle$ (blue), $\left\langle\omega \partial_{p} s\right\rangle$ (red), and $\left\langle\omega \partial_{p} q\right\rangle$ (green) for the boxes in Fig. 1 from the CESM (solid) and ERA-Interim (crosshatched). 
To explore the causes responsible for the different vertical MSE advection trends between the western and eastern Pacific ITCZs, we further decompose the vertical MSE advection term into dry static energy $\left\langle\omega \partial_{p} s\right\rangle$ and moisture $\left\langle\omega \partial_{p} q\right\rangle$ parts (Fig. 3b). The effects of the last two terms are competing with each other, as mentioned in Yu et al. (1998). In the western Pacific ITCZ, the magnitude of $\left\langle\omega \partial_{p} s\right\rangle$ is larger than that of $\left\langle\omega \partial_{p} q\right\rangle$ because of a greater top-heavy structure of vertical motion, resulting in a positive value of the vertical MSE advection. On the other hand, the magnitude of $\left\langle\omega \partial_{p} q\right\rangle$ is larger than that of $\left\langle\omega \partial_{p} s\right\rangle$ in the eastern Pacific, resulting in a negative value of the vertical MSE advection. We note that the vertical dry static energy advection is always positive and comparable in size in the two domains. Therefore, it is the vertical moisture transport that determines the sign of vertical MSE advection. Because the moisture is confined mostly in the lower troposphere, the bottom-heavy structure of vertical motion in the eastern Pacific tends to produce a greater negative of $\left\langle\omega \partial_{p} q\right\rangle$ than that in the western Pacific with a top-heavy structure of vertical motion.

\section{Controlling factors for vertical MSE advection}

We now further examine how the different vertical structures of vertical motion control the sign and magnitude of vertical MSE advection based on the simple linear Reynolds decomposition, which includes contributions from changes in dry static energy $s$, moisture $q$, and pressure velocity $\omega$. The sensitivity of the size of the vertical MSE advection to the upper bound of integration is also examined.

\section{a. Impacts of large-scale environment (thermodynamic effect)}

We evaluated the contribution from the large-scale environment for each domain. Let $s^{\prime}$ and $q^{\prime}$ be the departure from the corresponding temporal and spatial mean averaged over the entire tropics (i.e., $s^{\prime}=s-\bar{s}$ and $\left.q^{\prime}=q-\bar{q}\right)$. Thus, changes of the vertical MSE advection due to MSE perturbation can be written as

$$
\left\langle\bar{\omega} \partial_{p} h^{\prime}\right\rangle=\left\langle\bar{\omega} \partial_{p} s^{\prime}\right\rangle+\left\langle\bar{\omega} \partial_{p} q^{\prime}\right\rangle .
$$

Figure 4 shows the area averages of each term in (4) over both the western Pacific and the eastern Pacific ITCZs for the two datasets. For the western Pacific ITCZ, $\left\langle\bar{\omega} \partial_{p} h^{\prime}\right\rangle$ shows a negative value, and the magnitude is slightly larger than the one in the eastern Pacific ITCZ. The contribution of the dry static energy anomaly $\left\langle\bar{\omega} \partial_{p} s^{\prime}\right\rangle$ is very small compared to the contribution from the moisture anomaly $\left\langle\bar{\omega} \partial_{p} q^{\prime}\right\rangle$.

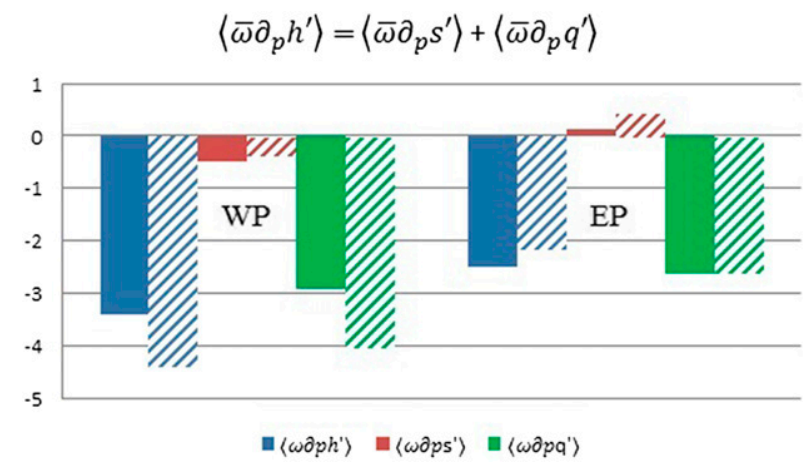

FIG. 4. As in Fig. 3, but for the averages of $\left\langle\bar{\omega} \partial_{p} h^{\prime}\right\rangle,\left\langle\bar{\omega} \partial_{p} s^{\prime}\right\rangle$, and $\left\langle\bar{\omega} \partial_{p} q^{\prime}\right\rangle$.

For the eastern Pacific ITCZ, $\left\langle\bar{\omega} \partial_{p} h^{\prime}\right\rangle$ also shows a negative value. The value of $\left\langle\bar{\omega} \partial_{p} s^{\prime}\right\rangle$ is slightly positive because of the colder SST in this region compared to other parts of the Pacific. A colder SST might introduce a negative sign of $\partial_{p} s^{\prime}$ that can reverse the sign of $\left\langle\bar{\omega} \partial_{p} s^{\prime}\right\rangle$ and prohibits the convection from occurring. This is similar to the mechanism for the threshold of tropical convection proposed by Johnson and Xie (2010). Except for magnitude differences, the estimated tendencies in the ERA-Interim analysis are very similar to those in the CESM simulation. In short, it is the moisture perturbation that contributes mostly to the negative value of $\left\langle\bar{\omega} \partial_{p} h^{\prime}\right\rangle$. However, one should also note that changes of vertical MSE advection due to MSE perturbation (thermodynamic effect) are very small compared to those due to changes of vertical motion (dynamic effect) discussed in the following subsection.

\section{b. Impacts of vertical velocity profile (dynamic effect)}

To examine the contribution from changes in $\omega$, a similar decomposition of the pressure velocity is applied, $\omega^{\prime}=\omega-\bar{\omega}$, which yields

$$
\left\langle\omega^{\prime} \partial_{p} \bar{h}\right\rangle=\left\langle\omega^{\prime} \partial_{p} \bar{s}\right\rangle+\left\langle\omega^{\prime} \partial_{p} \bar{q}\right\rangle .
$$

Figure 5 shows changes of the vertical MSE advection due to pressure velocity perturbation. For the western Pacific ITCZ, $\left\langle\omega^{\prime} \partial_{p} \bar{h}\right\rangle$ is positive because of the relative smaller magnitude of $\left\langle\omega^{\prime} \partial_{p} \bar{q}\right\rangle$ (negative contribution) than that of $\left\langle\omega^{\prime} \partial_{p} \bar{s}\right\rangle$ (positive contribution) for a more top-heavy structure of $\omega$. For the eastern Pacific ITCZ, $\left\langle\omega^{\prime} \partial_{p} \bar{h}\right\rangle$ is negative because of the greater magnitude of $\left\langle\omega^{\prime} \partial_{p} \bar{q}\right\rangle$ (negative contribution) than that of $\left\langle\omega^{\prime} \partial_{p} \bar{s}\right\rangle$ (positive contribution) for the more bottom-heavy structure of $\omega$. We also note that the patterns (including sign and size) shown in Fig. 5 are very similar to what we have shown in Fig. $3 b$. This clearly suggests that it is the structure of vertical motion (i.e., the dynamic effect) that determines the sign and size of the 


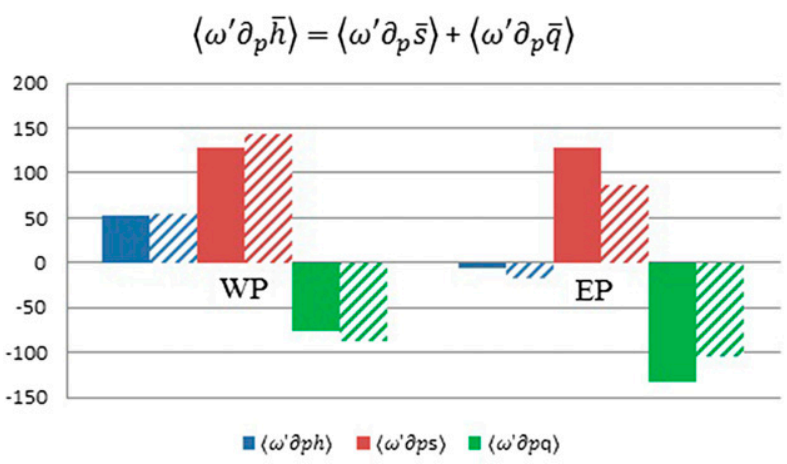

FIG. 5. As in Fig. 3, but for the averages of $\left\langle\omega^{\prime} \partial_{p} \bar{h}\right\rangle,\left\langle\omega^{\prime} \partial_{p} \bar{s}\right\rangle$, and $\left\langle\omega^{\prime} \partial_{p} \bar{q}\right\rangle$.

vertical MSE advection. In summary, a top-heavy convection structure favors a positive value of the vertical MSE advection and an export of column MSE, while a bottom-heavy convection structure favors a negative value of the vertical MSE advection and an import of column MSE.

To understand the background climatology of tropical convection, we demonstrate the spatial distribution of the maximum level of convection (MLC) in Fig. 6. The MLC represents the highest level that an air parcel can reach. It is defined as a pressure level at which the boundary layer saturation moist static energy intersects the environmental MSE curve, as in Yu et al. (1998). As shown, both the model simulation and reanalysis data show that the MLC is higher in the western Pacific than in the eastern Pacific. This is consistent with the fact that the value of vertical MSE advection tends to be positive in the western Pacific ITCZ and negative in the eastern Pacific ITCZ. This is because a higher (lower) MLC will contribute more (less) positive MSE flux, which determines the magnitude (and also the sign for what happens in the eastern Pacific) of vertical MSE advection because of a larger (smaller) contribution of MSE flux from the upper troposphere.

\section{c. Sensitivities to the upper bound of integration}

To understand whether the estimate of vertical MSE advection is sensitive to the choice of the upper bound of integration, four upper levels are tested: 200,150, 100, and $50 \mathrm{hPa}$. As shown in Fig. 7, while the sign of vertical MSE advection does not change, its magnitude is very sensitive to the choice of the upper bound of integration in the western Pacific ITCZ. The value of vertical MSE advection ranges from 28 to $40 \mathrm{~W} \mathrm{~m}^{-2}$ when the upper bound of integration changes from 200 to $50 \mathrm{hPa}$. On the other hand, the estimated vertical MSE advection shows almost no sensitivity to the choice of the upper bound of integration in the eastern Pacific ITCZ. The above analysis
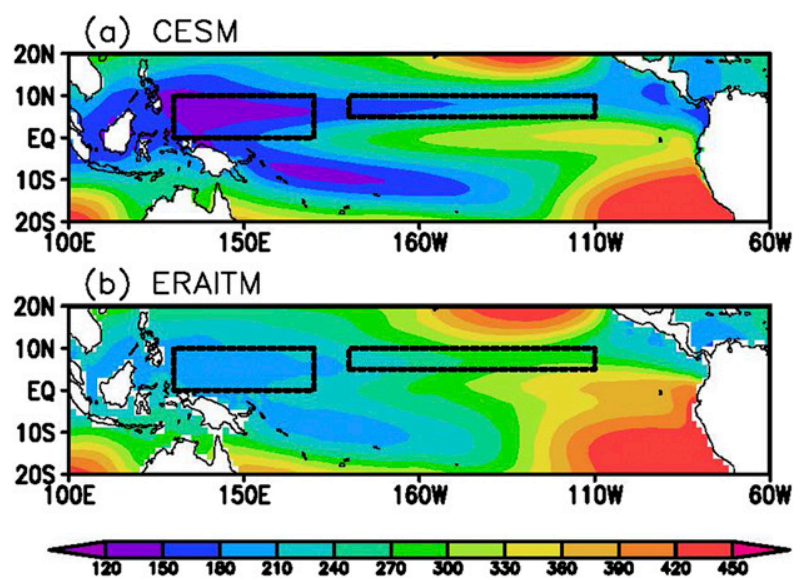

FIG. 6. The maximum level of convection (hPa) for the (a) CESM and (b) ERA-Interim.

shows that estimates of vertical MSE advection could be sensitive to the choice of the upper bound of integration. A fixed upper bound case might lead to spurious estimates of the vertical MSE advection, especially over the regions dominated by top-heavy vertical motion, such as the western Pacific ITCZ.

To understand the cause of the above sensitivity, we again decomposed the vertical MSE advection into contributions from dry static energy flux and moisture flux. As shown in Fig. 8, the dry static energy flux appears to dictate the changes of vertical MSE advection in the western Pacific ITCZ. When the upper bound of integration goes higher, the top-heavy structure of $\omega$ will add more weight from the upper troposphere, where the dry static energy is typically large. In contrast, the bottom-heavy structure in the eastern Pacific makes the estimates of MSE flux rather insensitive to the upper bound of integration. Besides, the moisture flux in both regions shows almost no sensitivity to the choice of upper bound (Fig. 8b) because the atmospheric moisture is confined mostly in the lower troposphere.

\section{$\left\langle\omega \partial_{p} h\right\rangle$}

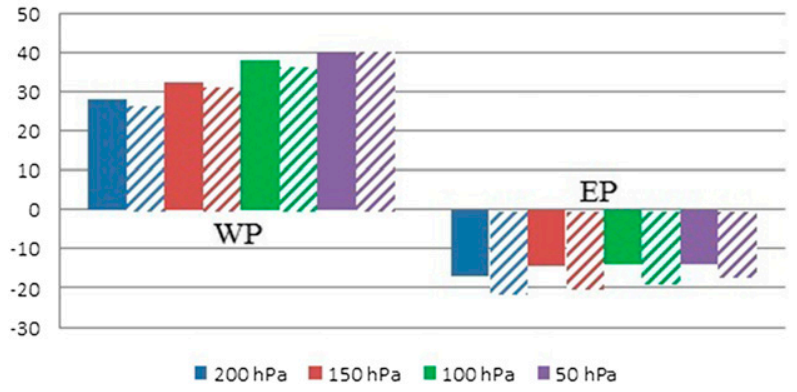

FIG. 7. Sensitivity to the upper bound $\left(\mathrm{W} \mathrm{m}^{-2}\right)$ of the vertical integration for the CESM (solid) and ERA-Interim (crosshatched) for different regions. 


$$
(a)\left\langle\omega \partial_{p} s\right\rangle
$$

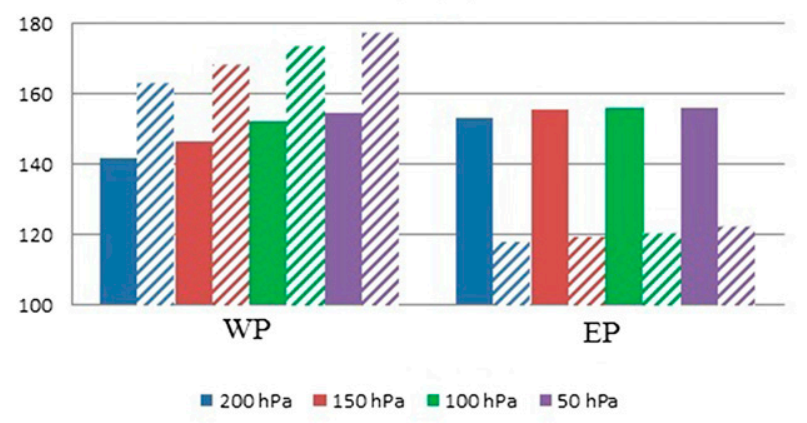

$(b)\left\langle\omega \partial_{p} q\right\rangle$

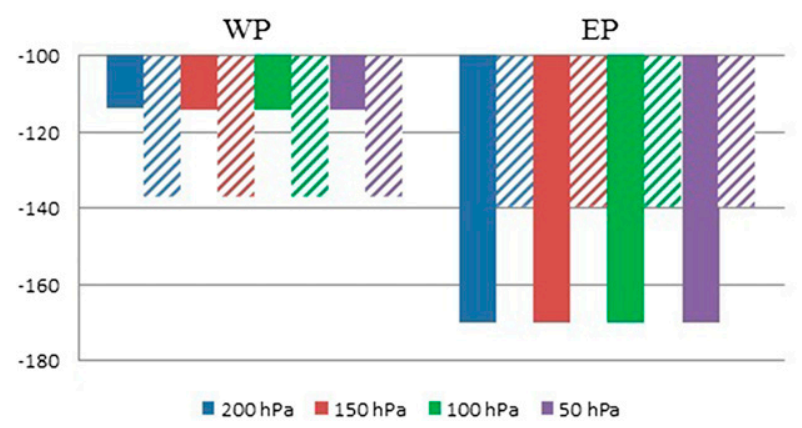

FIG. 8. As in Fig. 7, but for (a) $\left\langle\omega \partial_{p} s\right\rangle$ and (b) $\left\langle\omega \partial_{p} q\right\rangle$.

\section{Summary and discussion}

We have estimated the vertical moist static energy (MSE) advection for the western and eastern Pacific ITCZs using data from the ERA-Interim and the CESM simulation. It was found that the sign of vertical MSE advection is sensitive to the structure of vertical motion. For a top-heavy (bottom heavy) structure of vertical motion, such as the case occurring in the western (eastern) Pacific ITCZ, the value of vertical MSE advection tends to be positive (negative), implying an export (import) of the column MSE and a stabilization (destabilization) of the atmosphere. Factors controlling the above contrast are examined using a simple Reynolds decomposition of the MSE budget and an SVD analysis of MSE and $\omega$ fields, as shown in the appendix. Some notable findings are summarized as follows:

(i) A simple decomposition of the vertical MSE advection into vertical dry static energy and vertical moisture transports shows that the different signs of vertical MSE advection between the western and the eastern Pacific ITCZs is determined mainly by the vertical moisture transport rather than by the vertical dry static energy transport (see Fig. 3b).

(ii) A further decomposition of the vertical MSE advection into contributions from changes in dry static energy and moisture (referred to as the a)

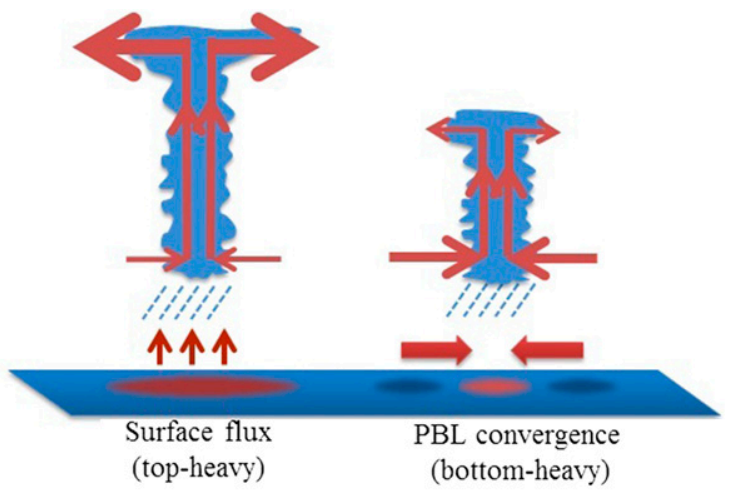

b)

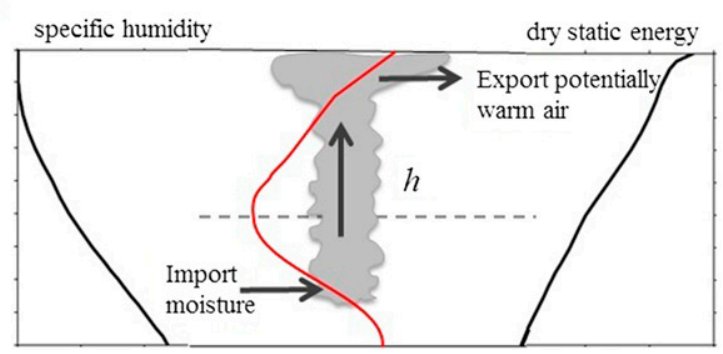

FIG. 9. A schematic diagram showing the different roles of shallow and deep convection (large-scale vertical motion) in venting static energy.

thermodynamic effect) and changes in vertical velocity (referred to as the dynamic effect) suggest that the size of vertical moisture advection is determined largely by the structure of vertical motion (i.e., by the $\left\langle\omega^{\prime} \partial_{p} \bar{q}\right\rangle$ term). A bottomheavy (top heavy) structure of vertical motion would produce a larger (smaller) negative value of $\omega^{\prime} \partial_{p} \bar{q}$ that favors a negative (positive) value of the vertical MSE advection (see Figs. 4, 5).

(iii) The magnitude of vertical MSE advection can be very sensitive to the upper bound of integration in regions dominated by top-heavy structures of convection, such as the case occurring in the western Pacific ITCZ. A larger value of vertical MSE advection occurs when a higher upper bound of integration is chosen. The estimates of vertical MSE advection show almost no sensitivity to the upper bound of integration in the eastern Pacific ITCZ, where the mean convection profile is dominated by a bottom-heavy structure (see Figs. 7, 8).

(iv) The SVD analysis indicates that a robust relation occurs between MSE and $\omega$ fields in both the western and the eastern Pacific ITCZs (see Fig. A1). The singular vectors of MSE and $\omega$ in the former region reveal the influence of a top-heavy structure of convection, while the singular vectors in the latter show the dominance of a bottom-heavy structure of convection (see Fig. A2). 

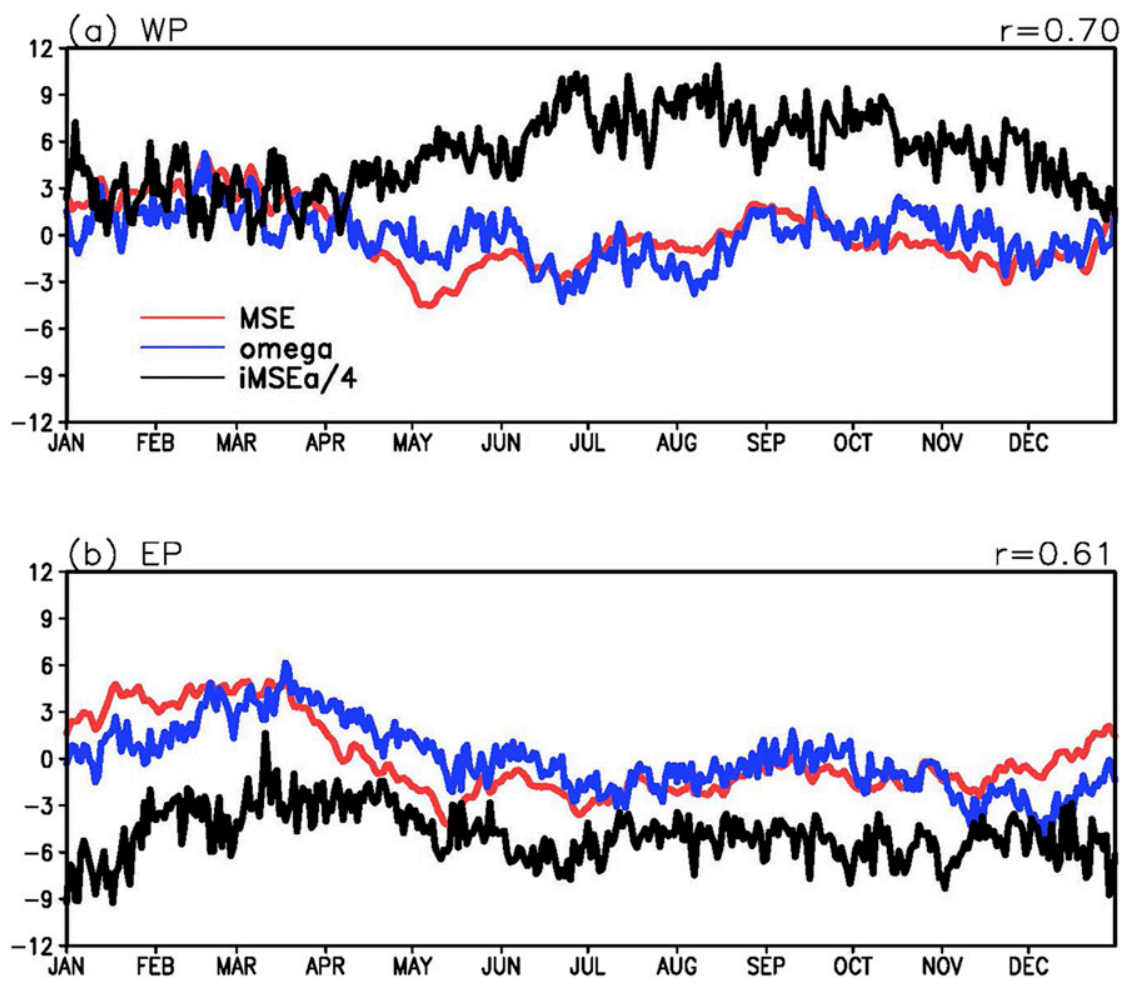

FIG. A1. Expansion coefficient (time series) of the leading SVD mode between the MSE (red) and $\omega$ (blue) profiles over (a) the western Pacific and (b) the eastern Pacific for the ERAInterim. The daily variation of the vertical MSE advection (black, with magnitude divided by 4) is also displayed for comparison. The correlation coefficient between MSE and $\omega$ time series is shown in the top-right corner of each panel.

The impact of the vertical structure of convection (large-scale vertical motion) on the sign of vertical MSE advection can be realized more easily using a schematic diagram (Fig. 9). In the tropics, the mean MSE profile typically features a minimum MSE in the midtroposphere, accompanied by larger MSE in the atmospheric boundary layer and the upper troposphere. The large MSE in the boundary layer is a result of abundant
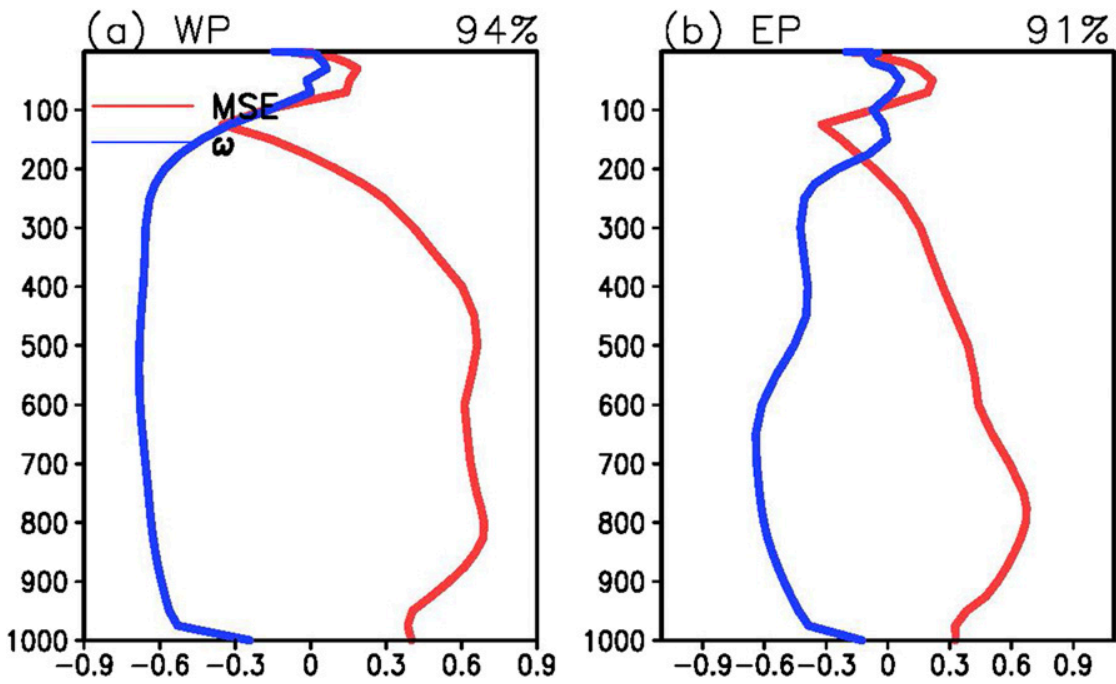

FIG. A2. The first SVD mode heterogeneous covariance profiles for the MSE (red) and $\omega$ (blue) from the ERA-Interim. 
moisture evaporated from warm tropical oceans, while the large MSE in the upper troposphere is associated with a rapidly increasing dry static energy (an equivalent increase in the potential temperature) below the tropopause. A top-heavy structure of vertical motion will put more weight on the upper-level divergence of MSE than its low-level counterpart (i.e., convergence of MSE), resulting in an export of MSE and a positive value of the vertical MSE advection. On the other hand, a bottomheavy structure of vertical motion will add extra weight to the low-level convergence of MSE compared to its upperlevel counterpart (i.e., divergence of MSE), resulting in an import of MSE and a negative value of the vertical MSE advection.

Although this study has shown that, in a climatological sense, the tropical western (eastern) Pacific ITCZ is dominated by top-heavy (bottom heavy) convection, it does not rule out the possible coexistence of different types of convection on a day-to-day evolution basis, such as the case demonstrated in the SVD analysis (see Fig. A2). One should also note that results shown here are not based on "genuine" observations, even for the ERAInterim data, as they are a blend of modeling and observations. More long-term in situ and satellite observations of cumulus cloud properties, such as cloud fraction, cloudtop pressure, and heating profile, are required to provide a statistic view of the partitioning between shallow and deep convection in the tropical areas.

Acknowledgments. The first author particularly thanks Trang Tran, Jenny Chang, and Bor-Ting Jong for useful discussions. Comments from Drs. Chia-Chi Wang, Pei-Hua Tan, and Wei-Liang Lee are greatly appreciated. This research is supported by the National Science Council under Grant NSC102-2111-M-001-002MY3. The second author (JYY) is supported by the Ministry of Science and Technology under Grant MOST103-2111-M-008-026-MY2. We thank the four anonymous reviewers for their useful comments and suggestions to improve this manuscript.

\section{APPENDIX}

\section{Day-to-Day Correlation between MSE and $\omega$ Profiles}

We analyze the seasonal cycle of vertical MSE advection, $\left\langle\omega \partial_{p} h\right\rangle$, and its association with $\omega$ and MSE on a daily temporal-resolution basis. Since we are interested in the common features of the MSE and $\omega$ profiles, the SVD analysis is used to examine the pattern of covariability between the two fields. For brevity, we only show the results from ERA-Interim data here.

\section{a. Temporal correlation}

The time series or expansion coefficients of MSE and $\omega$ fields for the leading SVD mode are shown in Fig. A1. In the western Pacific ITCZ, the first SVD mode accounts for about $94 \%$ of the squared covariance fraction, with a robust correlation coefficient of 0.70 between the time series of MSE and $\omega$ (Fig. A1a). Except for a few days in winter and spring, the values of vertical MSE advection are positive throughout the year, with larger magnitudes occurring in boreal summer, consistent with the results shown in section 3. In the eastern Pacific ITCZ, the first SVD mode accounts for about $91 \%$ of the square covariance fraction with a correlation coefficient of 0.61 (Fig. A1b). The values of vertical MSE advection are generally negative all yearround except for a few days in spring, also consistent with the results shown in section 3 .

The second SVD mode appears to be associated more with the second baroclinic mode. Since it only accounts for a small fraction of the total covariance, we omit its discussion here.

\section{b. Vertical correlation}

Figure A2 shows the singular vectors of MSE and $\omega$. These vertical profiles were formed from the so-called heterogeneous correlations (Wallace et al. 1992); for instance, the MSE heterogeneous correlation profile is the vector of correlation coefficients between the gridpoint values of the MSE field and the expansion coefficient of the $\omega$ field.

In the western Pacific ITCZ, the first singular vectors of MSE and $\omega$ show clear evidence of deep convection influence. The MSE exhibits positive anomalies in the troposphere with two local maxima: one at around $800 \mathrm{hPa}$ and the other at around $400 \mathrm{hPa}$. The vertical motion, on the other hand, shows ascending anomalies throughout the troposphere with a slightly top-heavy structure (Fig. A2a). In the eastern Pacific ITCZ, the first singular vectors of MSE and $\omega$ demonstrate the dominance of the bottom-heavy structure of convection. The MSE has a maximum in the lower troposphere at around $800 \mathrm{hPa}$, while the corresponding $\omega$ profile shows a maximum ascending at around $700 \mathrm{hPa}$ (Fig. A2b).

\section{REFERENCES}

Back, L. E., and C. S. Bretherton, 2006: Geographic variability in the export of moist static energy and vertical motion profiles in the tropical Pacific. Geophys. Res. Lett., 33, L17810, doi:10.1029/2006GL026672.

Bretherton, C. S., C. Smith, and J. M. Wallace, 1992: An intercomparison of methods for finding coupled patterns in climate data. 
J. Climate, 5, 541-560, doi:10.1175/1520-0442(1992)005<0541 $\mathrm{AIOMFF}>2.0 . \mathrm{CO} ; 2$.

— M. E. Peters, and L. E. Back, 2004: Relationships between water vapor path and precipitation over the tropical oceans. J. Climate, 17, 1517-1528, doi:10.1175/1520-0442(2004)017<1517: RBWVPA $>2.0 . \mathrm{CO} ; 2$.

Chen, C.-A., J.-Y. Yu, and C. Chou, 2016: Impacts of vertical structure of convection in global warming: The role of shallow convection. J. Climate, 29, 4665-4684, doi:10.1175/JCLI-D-15-0563.1.

Cherry, S., 1996: Singular value decomposition analysis and canonical correlation analysis. J. Climate, 9, 2003-2009, doi:10.1175/1520-0442(1996)009<2003:SVDAAC >2.0.CO;2.

_ 1997: Some comments on singular value decomposition analysis. J. Climate, 10, 1759-1761, doi:10.1175/1520-0442(1997)010<1759: SCOSVD $>2.0 . \mathrm{CO} ; 2$.

Chou, C., L.-F. Huang, L. Tseng, J.-Y. Tu, and P.-H. Tan, 2009a: Annual cycle of rainfall in the western North Pacific and East Asian sector. J. Climate, 22, 2073-2094, doi:10.1175/2008JCLI2538.1.

— J. D. Neelin, C.-A. Chen, and J.-Y. Tu, 2009b: Evaluating the rich-get-richer mechanism in tropical precipitation change under global warming. J. Climate, 22, 1982-2005, doi:10.1175/ 2008JCLI2471.1.

— , T.-C. Wu, and P.-H. Tan, 2013: Changes in gross moist stability in the tropics under global warming. Climate Dyn., $\mathbf{4 1}$ 2481-2496, doi:10.1007/s00382-013-1703-2.

Dee, D. P., and Coauthors, 2011: The ERA-Interim reanalysis: Configuration and performance of the data assimilation system. Quart. J. Roy. Meteor. Soc., 137, 553-597, doi:10.1002/qj.828.

Hannah, W. M., and E. D. Maloney, 2014: The moist static energy budget in NCAR CAM5 hindcasts during DYNAMO. J. $A d v$. Model. Earth Syst., 6, 420-440, doi:10.1002/2013MS000272.

Johnson, N. C., and S.-P. Xie, 2010: Changes in the sea surface temperature threshold for tropical convection. Nat. Geosci., $\mathbf{3}$, 842-845, doi:10.1038/ngeo1008.

Lilly, D. K., 1968: Models of cloud-topped mixed layers under a strong inversion. Quart. J. Roy. Meteor. Soc., 94, 292-309, doi:10.1002/qj.49709440106.

Lindzen, R. S., and S. Nigam, 1987: On the role of sea surface temperature gradients in forcing low-level winds and convergence in the tropics. J. Atmos. Sci., 44, 2418-2436, doi:10.1175/ 1520-0469(1987)044<2418:OTROSS > 2.0.CO;2.

Ma, Z., J. Fei, X. Huang, and X. Cheng, 2015: A potential problem with the application of moist static energy in tropical cyclone studies. J. Atmos. Sci., 72, 3009-3019, doi:10.1175/JAS-D-14-0367.1.

Maloney, E. D., A. H. Sobel, and W. M. Hannah, 2010: Intraseasonal variability in an aquaplanet general circulation model. $J . A d v$. Model. Earth Syst., 2 (5), doi:10.3894/JAMES.2010.2.5.

Neelin, J. D., and I. M. Held, 1987: Modeling tropical convergence based on the moist static energy budget. Mon. Wea. Rev., 115 3-12, doi:10.1175/1520-0493(1987)115<0003:MTCBOT>2.0.CO;2.

_ vective adjustment and the Madden-Julian oscillation. Part I Analytical theory. J. Atmos. Sci., 51, 1876-1894, doi:10.1175/ 1520-0469(1994)051<1876:MOTVUC>2.0.CO;2.

_ model-Formulation. J. Atmos. Sci., 57, 1741-1766, doi:10.1175/ 1520-0469(2000)057<1741:AQETCM>2.0.CO;2.
Neggers, R. A. J., J. D. Neelin, and B. Stevens, 2007: Impact mechanisms of shallow cumulus convection on tropical climate dynamics. J. Climate, 20, 2623-2642, doi:10.1175/ JCLI4079.1.

Newman, M., and P. D. Sardeshmukh, 1995: A caveat concerning singular value decomposition. J. Climate, 8, 352-360, doi:10.1175/1520-0442(1995)008<0352:ACCSVD>2.0.CO;2.

Ogura, Y., and N. A. Phillips, 1962: Scale analysis of deep and shallow convection in the atmosphere. J. Atmos. Sci., 19, 173-179, doi:10.1175/1520-0469(1962)019<0173:SAODAS > 2.0.CO;2.

Peters, M. E., Z. Kuang, and C. C. Walker, 2008: Analysis of atmospheric energy transport in ERA-40 and implications for simple models of the mean tropical circulation. J. Climate, 21, 5229-5241, doi:10.1175/2008JCLI2073.1.

Raymond, D. J., S. L. Sessions, A. H. Sobel, and Ž. Fuchs, 2009: The mechanics of gross moist stability. J. Adv. Model. Earth Syst., 1 (9), doi:10.3894/JAMES.2009.1.9.

Sobel, A. H., 2007: Simple models of ensemble-averaged precipitation and surface wind, given the sea surface temperature. The Global Circulation of the Atmosphere, T. Schneider and A. H. Sobel, Eds., Princeton University Press, 219-251.

Takayabu, Y. N., S. Shige, W.-K. Tao, and N. Hirota, 2010: Shallow and deep latent heating modes over tropical oceans observed with TRMM PR spectral latent heating data. J. Climate, 23, 2030-2046, doi:10.1175/2009JCLI3110.1.

Waite, M. L., and B. Khouider, 2010: The deepening of tropical convection by congestus preconditioning. J. Atmos. Sci., 67, 2601-2615, doi:10.1175/2010JAS3357.1.

Wallace, J. M., C. Smith, and C. S. Bretherton, 1992: Singular value decomposition of wintertime sea surface temperature and 500-mb height anomalies. J. Climate, 5, 561-576, doi:10.1175/ 1520-0442(1992)005<0561:SVDOWS $>2.0$. CO 2 .

Wang, Z., 2014: Role of cumulus congestus in tropical cyclone formation in a high-resolution numerical model simulation. J. Atmos. Sci., 71, 1681-1700, doi:10.1175/JAS-D-13-0257.1.

Wu, C.-M., B. Stevens, and A. Arakawa, 2009: What controls the transition from shallow to deep convection? J. Atmos. Sci., 66, 1793-1806, doi:10.1175/2008JAS2945.1.

Yanai, M., and R. Johnson, 1993: Impacts of cumulus convection on thermodynamic fields. The Representation of Cumulus Convection in Numerical Models, Meteor. Monogr., No. 46, Amer. Meteor. Soc., 39-62, doi:10.1007/978-1-935704-13-3_4.

Yano, J.-I., and R. Plant, 2012: Interactions between shallow and deep convection under a finite departure from convective quasi equilibrium. J. Atmos. Sci., 69, 3463-3470, doi:10.1175/ JAS-D-12-0108.1.

Yu, J.-Y., and J. D. Neelin, 1994: Modes of tropical variability under convective adjustment and the Madden-Julian oscillation. Part II: Numerical results. J. Atmos. Sci., 51, 1895-1914, doi:10.1175/1520-0469(1994)051<1895:MOTVUC>2.0.CO;2. , and _ 1997: Analytic approximations for moist convectively adjusted regions. J. Atmos. Sci., 54, 1054-1063, doi:10.1175/1520-0469(1997)054<1054:AAFMCA>2.0.CO;2.

, C. Chou, and J. D. Neelin, 1998: Estimating the gross moist stability of the tropical atmosphere. J. Atmos. Sci., 55, 1354-1372, doi:10.1175/1520-0469(1998)055<1354:ETGMSO>2.0.CO;2. 